\title{
Multiplicity results for a class of fractional differential equations with impulse
}

\author{
Yulin Zhao ${ }^{1}$, Xiaoyan Shi ${ }^{1 *}$ and Haibo Chen ${ }^{2}$
}

${ }^{\text {*Correspondence: }}$

xiaoyshi08@126.com

'School of Science, Hunan

University of Technology, Zhuzhou,

P.R. China

Full list of author information is

available at the end of the article

\begin{abstract}
In this paper, we apply Morse theory, local linking arguments and the Clark theorem to a study of the multiplicity of nontrivial solutions for a class of impulsive fractional differential equations with Dirichlet boundary conditions.
\end{abstract}

MSC: 26A33; 35A15; 34A08; 58E05

Keywords: Fractional differential equation; Nontrivial solution; Morse theory; Impulsive effects

\section{Introduction}

This paper focuses on the following fractional differential equations with impulsive effects:

$$
\left\{\begin{array}{l}
{ }_{t} D_{T}^{\alpha}\left({ }_{0}^{c} D_{t}^{\alpha} u(t)\right)+\kappa(t) u(t)=f(t, u(t)), \quad 0<t<T, t \neq t_{j}, \\
\left.\Delta\left({ }_{t} D_{T}^{\alpha-1}{ }_{0}^{c} D_{t}^{\alpha} u\right)\right)\left(t_{j}\right)=I_{j}\left(u\left(t_{j}\right)\right), \quad j=1,2, \ldots, m, \\
u(0)=u(T)=0,
\end{array}\right.
$$

where $0<\alpha \leq 1,{ }_{0}^{c} D_{t}^{\alpha}$ and ${ }_{t} D_{T}^{\alpha}$ denote the left Caputo fractional derivative and the right Riemann-Liouville fractional derivative of order $\alpha$, respectively, $0=t_{0}<t_{1}<\cdots<t_{m+1}=$ $T$, and $\Delta\left({ }_{t} D_{T}^{\alpha-1}\left({ }_{0}^{c} D_{t}^{\alpha} u\right)\right)\left(t_{j}\right)={ }_{t} D_{T}^{\alpha-1}\left({ }_{0}^{c} D_{t}^{\alpha} u\right)\left(t_{j}^{+}\right)-{ }_{t} D_{T}^{\alpha-1}\left({ }_{0}^{c} D_{t}^{\alpha} u\right)\left(t_{j}^{-}\right)$, where ${ }_{t} D_{T}^{\alpha-1}\left({ }_{0}^{c} D_{t}^{\alpha} u\right)\left(t_{j}^{+}\right)=$ $\lim _{t \rightarrow t_{j}^{+} t} D_{T}^{\alpha-1}\left({ }_{0}^{c} D_{t}^{\alpha} u\right)(t),{ }_{t} D_{T}^{\alpha-1}\left({ }_{0}^{c} D_{t}^{\alpha} u\right)\left(t_{j}^{-}\right)=\lim _{t \rightarrow t_{j}^{-}} D_{T}^{\alpha-1}\left({ }_{0}^{c} D_{t}^{\alpha} u\right)(t)$, and $f \in C([0, T] \times \mathbb{R})$, $I_{j} \in C(\mathbb{R}, \mathbb{R})$. For the function $\kappa$ we assume the following condition:

(H0): $\kappa \in C[0, T]$ and $\operatorname{essinf}_{t \in[0, T]} \kappa(t)=\kappa_{0}>-\lambda_{1}$, where $\lambda_{1}$ is the first eigenvalue of the operator ${ }_{t} D_{T}^{\alpha}\left({ }_{0}^{c} D_{t}^{\alpha} u(t)\right)$ (see (1.4)).

Fractional differential equations (FDEs for short) have been recently proved to be strong tools in the modeling of many phenomena in various areas of science as physics, biology, geology, polymer rheology, control theory, chemistry and engineering, etc. There have been important advances in the theory of fractional calculus and fractional ordinary and partial differential equations recently, for more details on fractional calculus theory, see the monographs of Podlubny [1], Kilbas et al. [2], Zhou [3] and the references therein. Based on variational methods and critical point theory, Jiao and Zhou [4] investigated the existence of at least one weak solution to the following nonlinear FDEs:

$$
\left\{\begin{array}{l}
{ }_{t} D_{T}^{\alpha}\left({ }_{0} D_{t}^{\alpha} u(t)\right)=\nabla F(t, u(t)), \quad \text { a.e. } t \in[0, T], \\
u(0)=u(T)=0,
\end{array}\right.
$$

(c) The Author(s) 2018. This article is distributed under the terms of the Creative Commons Attribution 4.0 International License (http://creativecommons.org/licenses/by/4.0/), which permits unrestricted use, distribution, and reproduction in any medium, provided you give appropriate credit to the original author(s) and the source, provide a link to the Creative Commons license, and indicate if changes were made. 
where $\alpha \in(0,1],{ }_{0} D_{t}^{\alpha}$ and ${ }_{t} D_{T}^{\alpha}$ are the left and right Riemann-Liouville fractional derivatives respectively. $F:[0, T] \times \mathbf{R}^{N} \rightarrow \mathbf{R}$ (with $N \geq 1$ ) is an appropriate given function and $\nabla F(t, u)$ is the gradient of $F$ at $u$. Since then the variational methods were used to deal with the existence of solutions for fractional differential equations by many authors. In addition, impulsive problems for differential equations appear in mathematical models with sudden and discontinuously changes of their states in pharmacology, population dynamics, optimal control, etc. [5]. Very recently, Bonanno et al. [6], Fečkan et al. [7] and D'Aguì et al. [8] have investigated the existence results of solutions for impulsive FDEs. For more related work on impulsive FDEs, we refer the interested reader to [9-18] and the references therein. However, the main techniques used in these references are the coincidence degree theory, fixed-point theorems and critical point theory in nonlinear analysis but not Morse theory.

On the other hand, in the last few years, Morse theory has been recently used to deal with the existence of solutions for impulsive differential equations [19-22] having the corresponding variational structure. In [21], Shi and Chen considered the following impulsive boundary value problems:

$$
\left\{\begin{array}{l}
-u^{\prime \prime}(t)=f(t, u), \quad t \in[0, T] \backslash\left\{t_{1}, t_{2}, \ldots, t_{m}\right\} \\
\triangle u^{\prime}\left(t_{j}\right)=I_{j}\left(u\left(t_{j}\right)\right), \quad j=1,2, \ldots, m, \\
u(0)=u(T)=0 .
\end{array}\right.
$$

Under some natural assumptions, the authors established some new results on the existence of three nontrivial solutions for problems (1.2) via Morse theory. However, to the best of our knowledge, with exception of [23], little work has been done on the existence and multiplicity of solutions for impulsive FDEs with Dirichlet boundary conditions by using Morse theory. More precisely, in [23], the authors studied problem (1.1) and obtained the existence of one nontrivial solution via computing the critical groups at infinity and at zero. Motivated by the above described work, we are interested in investigating the multiplicity of nontrivial solutions for problem (1.1) via Morse theory, local linking arguments and the Clark theorem.

A number $\lambda \in \mathbb{R}$ is said to be an eigenvalue of the following Dirichlet problem:

$$
\left\{\begin{array}{l}
{ }_{t} D_{T}^{\alpha}\left({ }_{0}^{c} D_{t}^{\alpha} u(t)\right)=\lambda u(t), \\
u(0)=u(T)=0,
\end{array}\right.
$$

if there exists a nontrivial solution $u \in E_{0}^{\alpha}\left(E_{0}^{\alpha}\right.$ be given in Definition 2.1) such that

$$
\int_{0}^{T}{ }_{0}^{c} D_{t}^{\alpha} u(t){ }_{0}^{c} D_{t}^{\alpha} v(t) d t-\lambda \int_{0}^{T} u(t) v(t) d t=0
$$

for any $v \in E_{0}^{\alpha}$. By Riesz-Schauder theory, we can show that problem (1.3) has a sequence of positive eigenvalues $\left\{\lambda_{k}\right\}_{k=1}^{\infty}$, and $\lambda_{1}$ can be characterized as

$$
\lambda_{1}=\inf _{u \in E_{0}^{\alpha} \backslash\{0\}} \frac{\int_{0}^{T}\left|{ }_{0}^{c} D_{t}^{\alpha} u(t)\right|^{2} d t}{\int_{0}^{T}|u(t)|^{2} d t} .
$$


Before stating our main results, we give the following hypotheses on the nonlinearity $f$ and $I_{j}(j=1,2, \ldots, m)$ :

(H1) For every $j$, there exist constants $a_{j}, b_{j}>0$ and $\gamma_{j} \in[0,1)$ such that $\left|I_{j}(u)\right| \leq a_{j}|u|^{\gamma_{j}}+$ $b_{j}$, and $I_{j}$ satisfy $\int_{0}^{u} I_{j}(s) d s \geq 0$ for all $u \in \mathbb{R}$.

(H2) There exist $c_{0}>0, q \geq 1$ such that $|f(t, u)| \leq c_{0}\left(1+|u|^{q-1}\right)$, a.e. $t \in[0, T], u \in \mathbb{R}$.

(H3) $\lim \sup _{|u| \rightarrow \infty} \frac{F(t, u)}{|u|^{2}}<\frac{1}{2}\left(\lambda_{1}+\kappa_{0}\right)$ uniformly on $t \in[0, T]$, where $F(t, u)=\int_{0}^{u} f(t, s) d s$.

(H4) There exist $r>0$ small and $c_{1}, c_{2}>0$ with $c_{2} \xi_{*}^{2}<1,0<\mu<\min \left\{\gamma_{j}+1\right\}$ such that

$$
c_{1}|u|^{\mu} \leq F(t, u) \leq \frac{c_{2}}{2}|u|^{2}, \quad|u| \leq r, t \in[0, T],
$$

where $\xi_{*}$ will be given in Remark A.

(H5) $f(t, u)$ and $I_{j}(u)(j=1,2, \ldots, m)$ are odd with respect to $u$. Now we state the main results of this paper.

Theorem 1.1 Assume conditions (H0)-(H4) hold, then the problem (1.1) has at least two nontrivial weak solutions in $E_{0}^{\alpha}$.

Theorem 1.2 Assume conditions (H0)-(H5) hold, then the problem (1.1) possesses at least $k$ distinct pairs of nontrivial weak solution in $E_{0}^{\alpha}$.

The paper is arranged as follows. The variational setting and some preliminaries are presented in Sect. 2 In Sect. 3, we complete the proof of Theorems 1.1 and 1.2.

\section{Variational setting and preliminaries}

For any fixed $t \in[0, T]$ and $1 \leq p<\infty$, let

$$
\begin{aligned}
& \|x\|_{\infty}=\max _{[0, T]}|x(t)|, \quad\|x\|_{L^{p}[0, t]}=\left(\int_{0}^{t}|x(s)|^{p} d s\right)^{1 / p} \text { and } \\
& \|x\|_{L^{p}}=\left(\int_{0}^{T}|x(t)|^{p} d t\right)^{1 / p} .
\end{aligned}
$$

In order to establish a variational structure for problem (1.1), we need to construct suitable function spaces. Denote by $C_{0}^{\infty}([0, T], \mathbb{R})$ the set of all functions $u \in C^{\infty}([0, T], \mathbb{R})$ with $u(0)=u(T)=0$. From Lemma 5.1 in [3], for any $u \in C_{0}^{\infty}([0, T], \mathbb{R})$ and $1<p<\infty$, we have $u \in L^{p}([0, T], \mathbb{R})$ and ${ }_{0}^{c} D_{t}^{\alpha} u \in L^{p}([0, T], \mathbb{R})$.

Definition 2.1 Let $0<\alpha \leq 1$. The fractional derivative space $E_{0}^{\alpha}$ is defined by the closure of $C_{0}^{\infty}([0, T], \mathbb{R})$, that is $E_{0}^{\alpha}=\overline{C_{0}^{\infty}([0, T], \mathbb{R})}$ with respect to the norm

$$
\|u\|_{\alpha, 2}=\left(\int_{0}^{T}\left|{ }_{0}^{c} D_{t}^{\alpha} u(t)\right|^{2} d t+\int_{0}^{T}|u(t)|^{2} d t\right)^{1 / 2}, \quad \forall u \in E_{0}^{\alpha}
$$

From Proposition 5.4 in [3], one finds that, for $0<\alpha \leq 1$, the space $E_{0}^{\alpha}$ is a reflexive and separable Banach space. We present some properties of the fractional derivative space $E_{0}^{\alpha}$.

Lemma 2.1 ([4] Let $0<\alpha \leq 1,\left\|_{0}^{c} D_{t}^{\alpha} u\right\|_{L^{2}}:=\left(\int_{0}^{T}\left|{ }_{0}^{c} D_{t}^{\alpha} u(t)\right|^{2} d t\right)^{1 / 2}$. For any $u \in E_{0}^{\alpha}$, we have

$$
\|u\|_{L^{2}} \leq \frac{T^{\alpha}}{\Gamma(\alpha+1)}\left\|_{0}^{c} D_{t}^{\alpha} u\right\|_{L^{2}} .
$$


Moreover, if $\alpha>\frac{1}{2}$, then

$$
\|u\|_{\infty} \leq \frac{T^{\alpha-\frac{1}{2}}}{\Gamma(\alpha) \sqrt{2 \alpha-1}}\left\|_{0}^{c} D_{t}^{\alpha} u\right\|_{L^{2}} .
$$

According to (2.2) we may consider the fractional space $E_{0}^{\alpha}$ with the norm $\|u\|_{\alpha}=\left\|{ }_{0}^{c} D_{t}^{\alpha} u\right\|_{L^{2}}$, which is equivalent to (2.1).

Lemma 2.2 ([4]) Let $\frac{1}{2}<\alpha \leq 1$. Assume that the sequence $\left\{u_{n}\right\}$ converges weakly to $u$ in $E_{0}^{\alpha}$, i.e., $u_{n} \rightarrow u$. Then $\left\{u_{n}\right\}$ converges strongly to $u$ in $C([0, T], \mathbb{R})$, i.e., $\left\|u_{n}-u\right\|_{\infty} \rightarrow 0$, as $n \rightarrow \infty$.

Lemma 2.3 Assume that $0<\alpha \leq 1$, If $(H 0)$ holds, then the norm

$$
\|u\|=\left(\left.\left.\int_{0}^{T}\right|_{0} ^{c} D_{t}^{\alpha} u(t)\right|^{2} d t+\int_{0}^{T} \kappa(t)|u(t)|^{2} d t\right)^{1 / 2}, \quad \forall u \in E_{0}^{\alpha},
$$

and the norm $\|\cdot\|_{\alpha}$ are equivalent, namely, there exist two positive constants $\xi, \eta$ such that

$$
\xi\|u\|_{\alpha} \leq\|u\| \leq \eta\|u\|_{\alpha}, \quad \text { for any } u \in E_{0}^{\alpha} .
$$

Proof Since essinf $t_{t \in[0, T]} \kappa(t)>-\lambda_{1}$, there exists $\varepsilon \in(0,1)$ such that $-\kappa_{0} \leq \lambda_{1}(1-\varepsilon)$. By virtue of (1.4), we get

$$
(1-\varepsilon) \int_{0}^{T}\left|{ }_{0}^{c} D_{t}^{\alpha} u(t)\right|^{2} d t \geq \lambda_{1}(1-\varepsilon) \int_{0}^{T}|u(t)|^{2} d t \geq-\kappa_{0} \int_{0}^{T}|u(t)|^{2} d t
$$

for any $u \in E_{0}^{\alpha}$. Therefore, for all $u \in E_{0}^{\alpha}$, we have

$$
\|u\|^{2} \geq \int_{0}^{T}\left(\left.||_{0}^{c} D_{t}^{\alpha} u(t)\right|^{2}+\kappa_{0}|u(t)|^{2}\right) d t \geq \varepsilon \int_{0}^{T}\left|{ }_{0}^{c} D_{t}^{\alpha} u(t)\right|^{2} d t=\varepsilon\|u\|_{\alpha}^{2}
$$

On the other hand, from (1.4) and (2.4) we deduce that

$$
\begin{aligned}
\|u\|^{2} & \leq \int_{0}^{T}\left(\left|{ }_{0}^{c} D_{t}^{\alpha} u(t)\right|^{2}+\|\kappa\|_{\infty}|u(t)|^{2}\right) d t \leq\left(1+\frac{\|\kappa\|_{\infty}}{\lambda_{1}}\right) \int_{0}^{T}\left|{ }_{0}^{c} D_{t}^{\alpha} u(t)\right|^{2} d t \\
& =\left(1+\frac{\|\kappa\|_{\infty}}{\lambda_{1}}\right)\|u\|_{\alpha}^{2} .
\end{aligned}
$$

Thus the norm $\|\cdot\|$ and the norm $\|\cdot\|_{\alpha}$ are equivalent and we may choose, for instance, $\xi=\sqrt{\varepsilon}$ and $\eta=\sqrt{1+\frac{\|\kappa\|_{\infty}}{\lambda_{1}}}$. In the sequel, we will consider $E_{0}^{\alpha}$ with the norm $\|\cdot\|$ defined in $(2.4)$.

$\operatorname{Remark} A$ From Lemmas 2.1 and 2.3, we have

(i) for $0<\alpha \leq 1,\|u\|_{L^{2}} \leq \frac{T^{\alpha}}{\Gamma(\alpha+1)}\|u\|_{\alpha} \leq \xi_{*}\|u\|$, where $\xi_{*}=\frac{T^{\alpha}}{\xi \Gamma(\alpha+1)}$;

(ii) for $\frac{1}{2}<\alpha \leq 1,\|u\|_{\infty} \leq \frac{T^{\alpha-\frac{1}{2}}}{\Gamma(\alpha) \sqrt{2 \alpha-1}}\|u\|_{\alpha} \leq \eta_{*}\|u\|$, where $\eta_{*}=\frac{T^{\alpha-\frac{1}{2}}}{\xi \Gamma(\alpha) \sqrt{2 \alpha-1}}$. 
Definition 2.2 We say that $u \in E_{0}^{\alpha}$ is a weak solution of problem (1.1) if the identity

$$
\int_{0}^{T}\left({ }_{0}^{c} D_{t}^{\alpha} u(t)_{0}^{c} D_{t}^{\alpha} v(t)+\kappa(t) u(t) v(t)\right) d t+\sum_{j=1}^{m} I_{j}\left(u\left(t_{j}\right)\right) v\left(t_{j}\right)=\int_{0}^{T} f(t, u(t)) v(t) d t
$$

holds for any $v \in E_{0}^{\alpha}$.

Consider the functional $\varphi: E_{0}^{\alpha} \rightarrow \mathbb{R}$ defined by

$$
\varphi(u)=\frac{1}{2}\|u\|^{2}+\sum_{j=1}^{m} \int_{0}^{u\left(t_{j}\right)} I_{j}(s) d s-\int_{0}^{T} F(t, u(t)) d t
$$

Since $f$ and $I_{j}(j=1, \ldots, m)$ are continuous, we can infer that $\varphi$ is continuous, differentiable and for all $u, v \in E_{0}^{\alpha}$

$$
\begin{aligned}
\left\langle\varphi^{\prime}(u), v\right\rangle= & \int_{0}^{T}\left({ }_{0}^{c} D_{t}^{\alpha} u(t)_{0}^{c} D_{t}^{\alpha} v(t)+\kappa(t) u(t) v(t)\right) d t+\sum_{j=1}^{m} I_{j}\left(u\left(t_{j}\right)\right) v\left(t_{j}\right) \\
& -\int_{0}^{T} f(t, u(t)) v(t) d t
\end{aligned}
$$

Then it is clear that the critical points of $\varphi$ are weak solutions of the problem (1.1).

Definition 2.3 We say that $\varphi$ satisfies the (PS) condition in $E_{0}^{\alpha}$, if any (PS) sequence $\left\{u_{n}\right\}_{n \in \mathbb{N}} \subset E_{0}^{\alpha}$, i.e., $\left\{\varphi\left(u_{n}\right)\right\}_{n \in \mathbb{N}}$ is bounded and $\varphi^{\prime}\left(u_{n}\right) \rightarrow 0$ as $n \rightarrow \infty$, has a strongly convergent subsequence in $E_{0}^{\alpha}$.

Definition 2.4 Let $E$ be a real Banach space and $\varphi \in C^{1}(E, \mathbb{R})$. Set $u$ be an isolated critical point of $\varphi$ with $\varphi(u)=c$, for $c \in \mathbb{R}$, and let $U$ be a neighborhood of $u$ such that $\varphi$ has only $u$ as a critical point in $U$. We call

$$
C_{k}(\varphi, u):=H_{k}\left(\varphi^{c} \cap U, \varphi^{c} \cap U \backslash\{u\}\right), \quad(k \in \mathbb{N}:=\{0,1,2, \ldots\})
$$

the $k$ th critical group of $\varphi$ at $u$, where $\varphi^{c}:=\{u \in E: \varphi(u) \leq c\}$ is the $c$-sublevel set, and $H_{k}$ is the singular relative homology group with coefficients in an Abelian group $G$. For more details, we refer the reader to [24].

One calls $u$ a homological nontrivial critical point of the functional $\varphi$ if at least one of the critical groups of $\varphi$ is nontrivial.

Lemma 2.4 ([25], Proposition 2.1) Let 0 be a critical point of $\varphi$ with $\varphi(0)=0$. Suppose that $\varphi$ has a local linking at 0 with respect to $E=V \oplus Y, k=\operatorname{dim} V<\infty$, that is, there exists $\rho>0$ small enough such that

$$
\varphi(u) \leq 0, \quad \forall u \in V,\|u\| \leq \rho \quad \text { and } \quad \varphi(u)>0, \quad \forall u \in Y, 0<\|u\| \leq \rho .
$$

Then $C_{k}(\varphi, 0) \nsucceq 0$, hence 0 is a homological nontrivial critical point of $\varphi$. 
Lemma 2.5 ([25], Theorem 2.1) Let $E$ be a real Banach space and let $\varphi \in C^{1}(E, \mathbb{R})$ satisfy the (PS)-condition and be bounded from below. If $\varphi$ has a critical point that is homological nontrivial and is not a minimizer of $\varphi$, then $\varphi$ has at least three critical points.

Lemma 2.6 ([26], Theorem 9.1) Let $E$ be a real Banach space, $\varphi \in C^{1}(E, \mathbb{R})$ with $\varphi$ even, bounded from below, and satisfying (PS)-condition. Suppose $\varphi(0)=0$, there is a set $\Omega \subset E$ such that $\Omega$ is homeomorphic to $S^{k-1}$ by an odd map, and $\sup _{\Omega} \varphi<0$. Then $\varphi$ possesses at least $k$ distinct pairs of critical points.

\section{Proofs of main results}

To obtain multiple nontrivial solutions of the problem (1.1), we shall present the following lemmas and compute the critical groups of the functional $\varphi$.

Lemma 3.1 Assume conditions (H0)-(H2) hold, then any bounded sequence $\left\{u_{n}\right\} \subset E_{0}^{\alpha}$ such that $\varphi^{\prime}\left(u_{n}\right) \rightarrow 0$ as $n \rightarrow \infty$ admits a strongly convergent subsequence in $E_{0}^{\alpha}$.

Proof Let $\left\{u_{n}\right\}$ be a bounded sequence in $E_{0}^{\alpha}$. Going to a subsequence if necessary, we can assume that $u_{n} \rightarrow u_{0}$ weakly in $E_{0}^{\alpha}$. Hence,

$$
u_{n} \rightarrow u_{0} \quad \text { in } L^{p}([0, T]), \quad u_{n} \rightarrow u_{0} \quad \text { a.e. } t \in[0, T] .
$$

We will show that $\left\{u_{n}\right\}$ converges strongly to $u_{0}$ in $E_{0}^{\alpha}$. Since $E_{0}^{\alpha}$ is reflexive space and $u_{n} \rightarrow u_{0}$ in $E_{0}^{\alpha}, \varphi^{\prime}\left(u_{n}\right) \rightarrow 0$ in $\left(E_{0}^{\alpha}\right)^{*}$, then from (2.5), we can obtain

$$
\left\langle\varphi^{\prime}\left(u_{n}\right)-\varphi^{\prime}\left(u_{0}\right), u_{n}-u_{0}\right\rangle \leq\left\|\varphi^{\prime}\left(u_{n}\right)\right\| \cdot\left\|u_{n}-u_{0}\right\|-\left\langle\varphi^{\prime}\left(u_{0}\right), u_{n}-u_{0}\right\rangle \rightarrow 0
$$

as $n \rightarrow \infty$. Moreover, it follows from (2.4) and Lemma 2.2 that $u_{n}$ is bounded in $C[0, T]$ and $\left\|u_{n}-u_{0}\right\|_{\infty} \rightarrow 0$ as $n \rightarrow \infty$. Hence, by (H1), (H2) and the Hölder inequality, we have

$$
\left\{\begin{array}{l}
\lim _{n \rightarrow \infty} \int_{0}^{T}\left(f\left(t, u_{n}(t)\right)-f\left(t, u_{0}(t)\right)\right)\left(u_{n}(t)-u_{0}(t)\right) d t=0, \\
\lim _{n \rightarrow \infty} \sum_{j=1}^{m}\left(I_{j}\left(u_{n}\left(t_{j}\right)\right)-I_{j}\left(t, u_{0}\left(t_{j}\right)\right)\right)\left(u_{n}\left(t_{j}\right)-u_{0}\left(t_{j}\right)\right)=0 .
\end{array}\right.
$$

Observe that

$$
\begin{aligned}
0 \leftarrow & \left\langle\varphi^{\prime}\left(u_{n}\right)-\varphi^{\prime}\left(u_{0}\right), u_{n}-u_{0}\right\rangle \\
= & \left\|u_{n}-u_{0}\right\|^{2}-\int_{0}^{T}\left[f\left(t, u_{n}\right)-f\left(t, u_{0}\right)\right]\left(u_{n}-u_{0}\right) d t \\
& +\sum_{j=1}^{m}\left[I_{j}\left(u_{n}\left(t_{j}\right)\right)-I_{j}\left(u_{0}\left(t_{j}\right)\right)\right]\left(u_{n}\left(t_{j}\right)-u_{0}\left(t_{j}\right)\right) \\
\geq & \left\|u_{n}-u_{0}\right\|^{2}-\left|\int_{0}^{T}\left[f\left(t, u_{n}\right)-f\left(t, u_{0}\right)\right] d t\right| \cdot\left\|u_{n}-u_{0}\right\|_{\infty} \\
& -\left|\sum_{j=1}^{m}\left[I_{j}\left(u_{n}\left(t_{j}\right)\right)-I_{j}\left(u_{0}\left(t_{j}\right)\right)\right]\right| \cdot\left\|u_{n}-u_{0}\right\|_{\infty} .
\end{aligned}
$$

Combining (3.2) and (3.3), we deduce that $\left\|u_{n}-u_{0}\right\|^{2} \rightarrow 0$ as $n \rightarrow \infty$, and we have $u_{n} \rightarrow u_{0}$ in $E_{0}^{\alpha}$. Thus, $\left\{u_{n}\right\}$ admits a convergent subsequence. This completes the proof. 
Lemma 3.2 Assume that (H0)-(H3) hold. Then $\varphi$ satisfies the (PS) condition.

Proof According to Lemma 3.1, it suffices to verify the boundedness of (PS) sequences. It follows from (H3) that, for some $\epsilon>0$ small, there exists a constant $C_{\epsilon}>0$ such that

$$
|F(t, u)| \leq \frac{1}{2}\left(\lambda_{1}+\kappa_{0}-\epsilon\right)|u|^{2}+C_{\epsilon}, \quad \text { for all } u \in \mathbb{R}, t \in[0, T]
$$

By assumption (H1) we get

$$
\int_{0}^{u\left(t_{j}\right)} I_{j}(s) d s \leq C_{j}|u|^{\gamma_{j}+1}, \quad \text { for some } C_{j}>0(j=1,2, \ldots, m)
$$

Thus, by means of (1.3), (3.4) and (3.5), for $u \in E_{0}^{\alpha}, u \neq 0$, we have

$$
\begin{aligned}
\varphi(u) \geq & \frac{1}{2} \int_{0}^{T}\left|{ }_{0}^{c} D_{t}^{\alpha} u(t)\right|^{2} d t+\frac{\kappa_{0}}{2} \int_{0}^{T}|u(t)|^{2} d t \\
& -\sum_{j=1}^{m} C_{j}|u|^{\gamma_{j}+1}-\frac{1}{2}\left(\lambda_{1}+\kappa_{0}-\epsilon\right) \int_{0}^{T}|u(t)|^{2} d t-C_{\epsilon} T \\
= & \frac{1}{2}\|u\|_{\alpha}^{2}-\frac{1}{2}\left(\lambda_{1}-\epsilon\right)\|u\|_{L^{2}}^{2}-\sum_{j=1}^{m} C_{j}|u|^{\gamma_{j}+1}-C_{\epsilon} T \\
\geq & \frac{1}{2}\|u\|_{\alpha}^{2}-\frac{1}{2 \lambda_{1}}\left(\lambda_{1}-\epsilon\right)\|u\|_{\alpha}^{2}-\sum_{j=1}^{m} C_{j}\left(\eta_{*}\right)^{\gamma_{j}+1}\|u\|^{\gamma_{j}+1}-C_{\epsilon} T \\
\geq & \frac{\epsilon}{2 \eta^{2}}\|u\|^{2}-\sum_{j=1}^{m} C_{j}^{*}\|u\|^{\gamma_{j}+1}-C_{\epsilon} T \rightarrow+\infty
\end{aligned}
$$

as $\|u\| \rightarrow \infty$, since $\eta>0,1 \leq \max \left\{\gamma_{j}+1\right\}<2$ and $C_{j}^{*}>0(j=1,2, \ldots, m)$ are positive constants. That is to say, $\varphi$ is coercive on $E_{0}^{\alpha}$. Therefore, we get the desired assertion.

Since $E_{0}^{\alpha}$ is a reflexive and separable Banach space, we may choose an orthogonal basis $\left\{e_{i}\right\}$ of $E_{0}^{\alpha}$ with $E_{0}^{\alpha}=\overline{\operatorname{span}\left\{e_{i}: i=1,2, \ldots\right\}}$. For $k=1,2, \ldots$, denote $X_{i}:=\operatorname{span}\left\{e_{i}\right\}, V_{k}=$ $\bigoplus_{i=1}^{k} X_{i}, Y_{k}=\overline{\bigoplus_{i=k}^{\infty} X_{i}}$, then $E_{0}^{\alpha}=V_{k} \oplus Y_{k}$.

Lemma 3.3 Assume that $(\mathrm{H} 0)-(\mathrm{H} 2)$ and $(\mathrm{H} 4)$ are satisfied. Then there exists $k_{0} \in \mathbb{N}$ such that $C_{k_{0}}(\varphi, 0) \neq 0$.

Proof According to Lemma 2.4, it suffices to show that the functional $\varphi$ has a local linking at zero with respect to $E_{0}^{\alpha}=V_{k} \oplus Y_{k}$.

Step 1: Set $u \in V_{k}$. Since $V$ is finite dimensional, from Remark A(ii) one sees that, for given $r$, there is $\rho \in(0,1]$ small such that, for $u \in V_{k},\|u\| \leq \rho$ implies $|u(t)| \leq r$ for $t \in$ $[0, T]$. Denote $F^{*}(t, u)=F(t, u)-c_{1}|u|^{\mu}$. Using $(\mathrm{H} 2)$ and (H4), there exists $c_{3}>0$ such that, for some $q>2$, we have $F^{*}(t, u) \geq-c_{3}|u|^{q}$ for all $t \in[0, T]$ and $|u|>0$. Therefore, for $u \in V_{k}$ with $\|u\| \leq \rho$, we get

$$
\varphi(u)=\frac{1}{2}\|u\|^{2}+\sum_{j=1}^{m} \int_{0}^{u\left(t_{j}\right)} I_{j}(s) d s-c_{1} \int_{0}^{T}|u|^{\mu} d t-\left(\int_{\{|u(t)| \leq r\}}+\int_{\{|u(t)|>r\}}\right) F^{*}(t, u(t)) d t
$$




$$
\begin{aligned}
& \leq \frac{1}{2}\|u\|^{2}+\sum_{j=1}^{m} C_{j}\left|u\left(t_{j}\right)\right|^{\gamma_{j}+1}-c_{1} \int_{0}^{T}|u|^{\mu} d t+c_{3} \int_{\{|u(t)|>r\}}|u|^{q} d t \\
& \leq \frac{1}{2}\|u\|^{2}+\sum_{j=1}^{m} C_{j}^{*}\|u\|^{\gamma_{j}+1}-c_{1}\|u\|_{L^{\mu}}^{\mu}+c_{4}\|u\|^{q},
\end{aligned}
$$

where $c_{4}$ and $C_{j}^{*}(j=1,2, \ldots, m)$ are positive constants. Notice that the norms on $V_{k}$ are equivalent to each other, thus $\|u\|_{L^{\mu}}$ is equivalent to $\|u\|$. Since $0<\mu<\min \left\{\gamma_{j}+1\right\} \leq$ $\max \left\{\gamma_{j}+1\right\}<2<q$, we have $\varphi(u) \leq 0$, for any $u \in V_{k}$ with $\|u\| \leq \rho$.

Step 2: Choose $u \in Y_{k}$, Due to the continuous embedding $E_{0}^{\alpha} \rightarrow C_{0}^{\infty}([0, T], \mathbb{R})$ is compact. Then, for given $r>0$, by (H2), (H4) and Young's inequality, there is a constant $c_{5}>0$ such that, for some $q>2$, one has

$$
|F(t, u)| \leq \frac{c_{2}}{2}|u|^{2}+c_{5}|u|^{q}, \quad \text { for all } t \in[0, T] \text { and }|u| \geq r .
$$

Denoting $H(t, u)=F(t, u)-\frac{c_{2}}{2}|u|^{2}$, from (H1), Remark A(i) and (3.6) we obtain

$$
\begin{aligned}
\varphi(u) & =\frac{1}{2}\|u\|^{2}+\sum_{j=1}^{m} \int_{0}^{u\left(t_{j}\right)} I_{j}(s) d s-\frac{c_{2}}{2} \int_{0}^{T}|u|^{2} d t-\left(\int_{\{|u(t)| \leq r\}}+\int_{\{|u(t)|>r\}}\right) H(t, u(t)) d t \\
& \geq \frac{1}{2}\left(1-c_{2} \xi_{*}^{2}\right)\|u\|^{2}-c_{5} \int_{\{\mid u(t)>r\}}|u(t)|^{q} d t \\
& \geq \frac{1}{2}\left(1-c_{2} \xi_{*}^{2}\right)\|u\|^{2}-c_{6}\|u\|^{q}
\end{aligned}
$$

where $c_{6}$ is positive constant. Since $c_{2} \xi_{*}^{2}<1, q \in(2,+\infty)$, we can show that $\varphi(u)>0$ as $u \in Y_{k}$ and $0<\|u\|<\rho$ with $\rho>0$ small enough. This completes the proof.

Proof of Theorem 1.1 By Lemma 3.2, $\varphi$ satisfies the (PS) condition and is bounded from below. According Lemma 3.3, the trivial solution $u=0$ is homological nontrivial and is not a minimizer. Then the conclusion follows immediately by Lemma 2.5 .

Proof of Theorem 1.2 From (H5), we can infer that $\varphi$ is even. Lemma 3.2 shows that $\varphi$ satisfies the (PS) condition and is bounded from below. For given $\rho>0$, set $\Omega=S_{\rho}=\{u \in$ $\left.V_{k}:\|u\|=\rho\right\}$. Therefore, just as shown in the proof of Lemma 3.3, if $\rho$ is small enough, we show that $\sup _{\Omega} \varphi(u)<0$. Obviously, $\operatorname{dim} V_{k}=k$, then it follows from Lemma 2.6 that $\varphi$ has at least $k$ distinct pairs of critical points. Thus, problem (1.1) has at least $k$ distinct pairs of nontrivial solutions.

\section{Conclusion}

In this paper, we have studied the existence and multiplicity of the solutions for an impulsive fractional differential equations with Dirichlet boundary conditions. Our approach is based on Morse theory, local linking arguments and the Clark theorem. In the future, we will consider the existence of solutions for the impulsive fractional differential equation with p-Laplacian via Morse theory. 


\section{Funding}

The third author was partially supported by the National Natural Science Foundation of China (No. 11671403).

\section{Availability of data and materials}

Not applicable.

\section{Competing interests}

The authors declare that they have no competing interests.

\section{Authors' contributions}

The authors declare that the study was realized in collaboration with the same responsibility. All authors read and approved the final manuscript.

\section{Author details}

${ }^{1}$ School of Science, Hunan University of Technology, Zhuzhou, P.R. China. ${ }^{2}$ School of Mathematics and Statistics, Central South University, Changsha, P.R. China.

\section{Publisher's Note}

Springer Nature remains neutral with regard to jurisdictional claims in published maps and institutional affiliations.

\section{Received: 27 March 2018 Accepted: 29 August 2018 Published online: 24 September 2018}

\section{References}

1. Podlubny, I.: Fractional Differential Equations. Academic Press, New York (1993)

2. Kilbas, A.A., Srivastava, H.M., Trujillo, J.J.: Theory and Applications of Fractional Differential Equations. North-Holland Mathematics Studies, vol. 204. Elsevier, Amsterdam (2006)

3. Zhou, Y.: Basic Theory of Fractional Differential Equations. World Scientific, Singapore (2014)

4. Jiao, F., Zhou, Y.: Existence results for fractional boundary value problem via critical point theory. Int. J. Bifurc. Chaos 22, $1250086(2012)$

5. Benchohra, M., Henderson, J., Ntouyas, S.: Theory of Impulsive Differential Equations, Contemporary Mathematics and Its Applications. Hindawi Publishing Corporation, New York (2006)

6. Bonanno, G., Rodríguez-López, R., Tersian, S.: Existence of solutions to boundary value problem for impulsive fractional differential equations. Fract. Calc. Appl. Anal. 17(3), 717-744 (2014)

7. Fečkan, M., Zhou, Y., Wang, J.: On the concept and existence of solution for impulsive fractional differential equations. Commun. Nonlinear Sci. Numer. Simul. 17, 3050-3060 (2012)

8. D’Aguì, G., Di Bella, B., Tersian, S.: Multiplicity results for superlinear boundary value problems with impulsive effects. Math. Methods Appl. Sci. 39, 1060-1068 (2016)

9. Heidarkhani, S., Zhao, Y., Caristi, G., Afrouz, G.A., Moradi, S.: Infinitely many solutions for perturbed impulsive fractional differential systems. Appl. Anal. 96(8), 1401-1424 (2017)

10. Zhao, Y., Tang, L.: Multiplicity results for impulsive fractional differential equations with p-Laplacian via variational methods. Bound. Value Probl. 2017, Article ID 123 (2017)

11. Rodríguez-López, R., Tersian, S.: Multiple solutions to boundary value problem for impulsive fractional differential equations. Fract. Calc. Appl. Anal. 17(4), 1016-1038 (2014)

12. Wang, J., Fečkan, M., Zhou, Y.: A survey on impulsive fractional differential equations. Fract. Calc. Appl. Anal. 19(4), 806-831 (2016)

13. Zhao, Y., Zhao, Y.: Nontrivial solutions for a class of perturbed fractional differential systems with impulsive effects. Bound. Value Probl. 2016, Article ID 129 (2016)

14. Torres, C., Nyamoradia, N.: Impulsive fractional boundary value problem with p-Laplace operator. J. Appl. Math. Comput. 55, 257-278 (2017)

15. Jiao, F., Zhou, Y.: Existence of solutions for a class of fractional boundary value problems via critical point theory. Comput. Math. Appl. 62, 1181-1199 (2011)

16. Nyamoradia, N., Rodríguez-López, R.: On boundary value problems for impulsive fractional differential equations. Appl. Math. Comput. 271, 874-892 (2015)

17. Nyamoradia, N., Rodríguez-López, R.: Multiplicity of solutions to fractional Hamiltonian systems with impulsive effects. Chaos Solitons Fractals 102, 254-263 (2017)

18. Torres, C.: Boundary value problem with fractional p-Laplacian operator. Adv. Nonlinear Anal. 5(2), 133-146 (2016)

19. Agarwal, R.P., Bhaskar, T.G., Perera, K.: Some results for impulsive problems via Morse theory. J. Math. Anal. Appl. 409, 752-759 (2014)

20. Ait-Mahiout, K., Djebali, S., Moussaoui, T.: Multiple solutions for an impulsive boundary value problem on the half-line via Morse theory. Topol. Methods Nonlinear Anal. 47(1), 219-240 (2016)

21. Shi, H., Chen, H.: Multiplicity results for a class of boundary value problems with impulsive effects. Math. Nachr. 289, 718-726 (2016)

22. Shi, H., Chen, H., Liu, H.: Morse theory and local linking for a class of boundary value problems with impulsive effects. J. Appl. Math. Comput. 51, 353-365 (2016)

23. Zhao, Y., Chen, H., Xu, C.: Nontrivial solutions for impulsive fractional differential equations via Morse theory. Appl. Math. Comput. 307, 170-179 (2017)

24. Chang, K.: Infinite Dimensional Morse Theory and Multiple Solution Problems. Birkhäuser, Boston (1993)

25. Liu, J., Su, J.: Remarks on multiple nontrivial solutions for quasi-linear resonant problems. J. Math. Anal. Appl. 258, 209-222 (2001)

26. Rabinowitz, P.H.: Minimax Methods in Critical Point Theory with Applications to Differential Equations. In CBMS Reg. Conf. Ser. Math., vol. 65. American Mathematical Society, Providence (1986) 